\title{
Nuevos grafitos descubiertos en Garb Asuán sur
}

\author{
Alejandro JIMÉNEZ SERRANO
}

El objetivo principal de la presente comunicación es dar a conocer preliminarmente un nuevo grupo de grafitos encontrados en Garb Asuán Sur, que fueron documentados durante una misión arqueológica que tuvo lugar en noviembre de 2005. Tal y como se podrá comprobar, los grafitos son diferentes desde el punto de vista cronológico y estilístico. Muchos de ellos datan del Periodo Predinástico, aunque también los hay de época faraónica e, incluso, posteriores. También se presentan y discuten otros hallazgos que, por el momento, no pueden adscribirse a un periodo en concreto.

\section{New graffiti discovered in South Garb Aswan}

The main aim of the present communication is to publish a preliminary study of a group of new graffiti found at Garb Aswan South during the Archaeological Mission carried out in November 2005. As will be seen, the range and the date of the graffiti are heterogeneous; many of them are dated to the Predynastic period, but others are Pharaonic and even later. We shall also report on and discuss some undated depictions.

KeY words: Qubbet-el Hawa, Aswan, Predynastic, Old Kingdom, Middle Kingdom, graffiti

$\mathrm{U}$ na de las áreas arqueológicas más conocidas de Egipto es Qubbet el-Hawa, donde fueron enterrados muchos de los más altos personajes del primer nomo del Alto Egipto desde finales del Reino Antiguo hasta el Reino Medio. Este yacimiento está incluido dentro de lo que actualmente denominamos como Garb Asuán. Las razones que nos han movido a incluir la necrópolis de los nobles bajo este nuevo nombre se basan en el hallazgo de nuevos grupos de tumbas que, sin duda, están relacionadas con las ya detectadas.
El proyecto estuvo dirigido por la Dra. Maysoum Al-Khouri, asistida por el Profesor Giuseppe C. Infranca, director del Istituto Superiore per le Tecniche di Conservazione dei Beni Culturali e dell'Ambiente "Antonino De Stefano", centro asociado al italiano CNR. Nuestra participación fue posible gracias a un convenio de colaboración firmado entre dicho instituto y la Universidad de Jaén. La Junta de Andalucía, dentro de su programa de Retorno de Doctores a Centros de Andalucía asumió los gastos de nuestra investigación.

* Queremos agradecer el interés y la ayuda científica que el Dr. M. el-Biali, inspector jefe del Supreme Council of Antiquities de la región de Asuán, ha mostrado durante nuestras investigaciones. Hemos de hacer partícipes de nuestros descubrimientos a los diferentes inspectores del Supreme Council of Antiquities que a diario nos acompañaron. También queremos agradecer la confianza que en nosotros ha depositado la directora de la presente misión la Dra. Maysoum Al-Khouri, así como las facilidades que siempre nos ha proporcionado el Prof. G. C. Infranca. Todos los dibujos con que se ilustra este trabajo han sido realizados por Juan Manuel Anguita Ordóñez.

Fecha de recepción: 16 de enero de 2007

Fecha de aceptación: 25 de junio de 2007 
Aunque el principal objetivo del proyecto en Garb Asuán es la conservación y la formación de técnicos egipcios, también destaca la intervención arqueológica, de la cual somos responsables junto con la Dra. Maysoum Al-Khouri ${ }^{1}$. Así, dentro de nuestras competencias, organizamos en noviembre de 2005 una prospección arqueológica en toda la necrópolis. Los resultados que a continuación presentamos sólo se refieren a una parte de todo el yacimiento, en concreto a Garb Asuán Sur.

Entendemos la zona arqueológica de Garb Asuán como el espacio comprendido entre

- Uadi Saman, al sur, al final del cual se alza el monasterio de San Simeón;

- el moderno pueblo de Garb Asuán, al norte;

- Uadi el-Deir, al oeste;

- el río Nilo, al este.

Más concretamente, Garb Asuán Sur se extendería entre Qubbet el-Hawa y Uadi Saman.

Dentro de esta gran área, la única zona estudiada exhaustivamente desde finales del siglo XIX ha sido el promontorio conocido como Qubbet el-Hawa ( $c f$. infra). Del resto de nuestra concesión que se extiende más al sur no hemos encontrado ningún documento o referencia, seguramente debido a la previsible mayor potencialidad arqueológica de Qubbet el-Hawa, al difícil acceso de la zona meridional y al riesgo que corrían otros yacimientos de la región (Ele[18 ] fantina, la ciudad de Asuán, File, las canteras de granito, etc.).

Por tanto, Garb Asuán Sur puede considerarse como una zona arqueológica completamente virgen que permitirá en el futuro realizar una investigación de los diferentes espacios, así como también una profundización de los procesos post-deposicionales, ignorados en la mayor parte de las investigaciones de campo desarrolladas hasta hace pocos años en Egipto.

\section{ESTUdIOS PIONEROS EN LA ZONA}

Desde finales del siglo XIX, se ha venido publicando una gran cantidad de trabajos de investigación sobre los monumentos de la región de Asuán, que abarcan con mayor o menor detalle los numerosos templos de la región, las canteras, estelas, tumbas y hasta los desarrollos históricos de algunos núcleos urbanos, entre los que destaca Elefantina.

Por lo que respecta al área de Garb Asuán, todas las publicaciones se han centrado en las tumbas de la necrópolis de Qubbet el-Hawa ${ }^{2}$ Desde las primeras noticias hasta los últimos descubrimientos no hace más de treinta años.

Junto con toda esta ingente cantidad de información, los petroglifos y las inscripciones también despertaron el interés de los pioneros de la Egiptología moderna. Así, en 1887, W. M. F. Petrie, publicó A Season in Egypt, en el cual el autor pretendía añadir, actualizar y contrastar parte de las inscripciones aparecidas con anterioridad en el Denkmäler de Lepsius, entre ellas muchas de la de la región de Asuán.

Estas obras compiladoras de material epigráfico fueron necesarias en esa época para crear un corpus textual que ayudara a reconstruir la Historia Antigua de Egipto. Poco después de la obra de Petrie, en 1894, apareció el primer tomo dedicado al Alto Egipto que J. de Morgan dirigió con el título de Catalogue de monuments

1. Los objetivos pueden consultarse en Al-Khouri, 2005.

2. Sobre los hallazgos arqueológicos, $c f$. Edel, 2008. 
et inscriptions de l'Égypte Antique. En ella, se recopiló una gran cantidad de documentos gráficos y escritos de todas las épocas grabados en la roca o en monumentos. Sin embargo, ni Petrie ni los compiladores del Catalogue de monuments se detuvieron en Garb Asuán Sur, probablemente por el ya comentado difícil acceso de la zona y por el ingente trabajo que tenían por delante que obligaba a centrarse en aquellas que corrían un mayor riesgo de destrucción o aquellas que sobresalían por su majestuoso carácter monumental o interés histórico. El caso es que una zona de más de $1 \mathrm{~km}$ de longitud fue obviada por estos investigadores ${ }^{3}$. Exactamente la misma carencia se puede observar en la obra publicada por Arthur Weigall en $1910^{4}$.

A pesar de los trabajos de campo que durante años llevaron a cabo investigadores tan reputados como Elmar Edel ${ }^{5}$ o Labib Habachi ${ }^{6}$ en la zona, estos grafitos nunca han sido publicados, por lo que hemos de considerar que nunca fueron conocidos por ningún miembro de la comunidad científica.

\section{LOS NUEVOS GRAFITOS}

El nuevo material que a continuación reproducimos fue documentado durante la última semana de noviembre de 2005 y se encuentra en general en malas condiciones debido al tipo de roca sedimentaria sobre la que fue grabado ${ }^{7}$. A pesar de ello, en la mayoría de los casos se puede determinar a grandes rasgos ante qué nos encontramos y, en algunos casos, incluso se puede determinar la época.

Todos los ejemplos presentados y estudiados a continuación han sido divididos teniendo en cuenta su condición de petroglifos, inscripciones o representaciones dudosas.

\section{Petroglifos}

\section{Petroglifo 1}

Dos órices o gacelas

Fecha: Predinástico

Figuras: 1a / 1b

Estos dos órices o gacelas datan sin ninguna duda del periodo Predinástico ${ }^{8}$, aunque debido al gran número de este tipo de representaciones durante la Prehistoria es imposible concretar la fase concreta 9 . Al menos uno de los animales (izquierda) parece estar domesticado, si interpretamos correctamente las trazas de lo que parece ser un collar ${ }^{10}$, lo que claramente indicaría su domesticación o su trascendencia religiosa.

Existen pocos ejemplos de animales representados de forma contrapuesta ${ }^{11}$. Su interpre-

3. Cf. de Morgan et alii, 1894: 141, cómo tras el estudio del monasterio de San Simeón se trata seguidamente la necrópolis de los nobles.

4. Cf. en Weigall, 1910, capítulo XX la ausencia de menciones de Garb Asuán Sur cuando trata la región de Asuán.

5. Edel, 2008.

6. Lamentablemente, Habachi sólo publicó alguna noticia sobre sus excavaciones en Qubbet el-Hawa en forma de algunos artículos dispersos, $c f$. Habachi, 1981. Afortunadamente, sus notas han sido incluidas en el estudio general que realizó Edel, 2008.

7. Sobre los aspectos geológicos de la rivera occidental de Asuán, $c f$. Heldal, Bøe y Müller, 2007: 51-67.

8. Cf. los paralelos en la Baja Nubia, Hellström, 1970: 95, pls. Corpus L165.

9. Resulta bastante probable que daten del periodo comprendidos entre las fases de Naqada I-II.

10. Las representaciones de animales (ganado vacuno y caprino) con collar es bastante común en época Predinástica, $c f$. Červíček, 1974: Abb. 61, 64, 105, 333, 343, 348, 351, 355, 384.

11. En el Desierto Oriental de la Baja Nubia, cf. Červíček, 1974: Abb. 317 (Magal Hodein). 


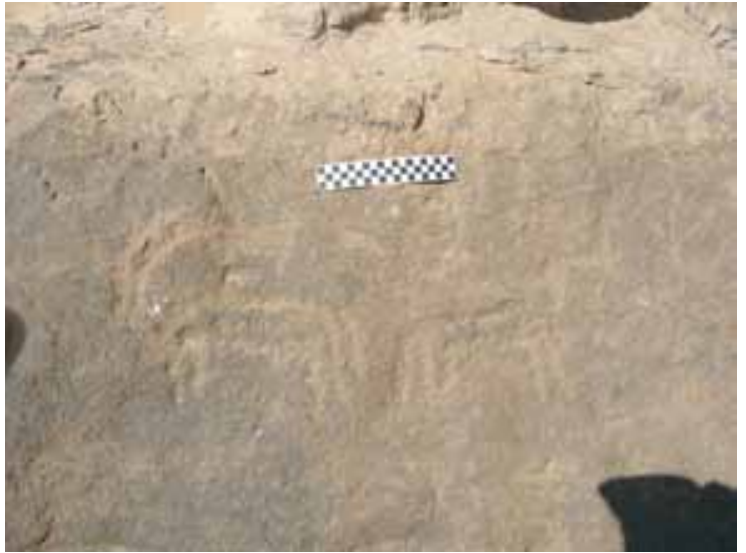

Figura 1a

tación se nos escapa. Pudo mostrar una escena natural; ejemplos de ellas no nos faltan, sobre todo en la vecina Nubia ${ }^{12}$. Aun así hemos de recordar a una hija de la diosa Satis, adorada posteriormente en la región que, era representada como una gacela ${ }^{13}$.

Petroglifo 2

Cocodrilo

Fecha: Predinástico

Figuras: 2a / 2b

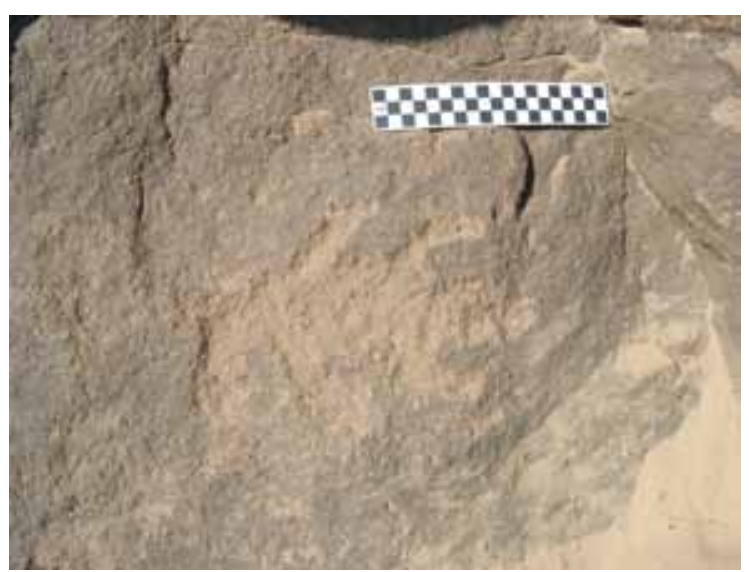

Figura $2 a$

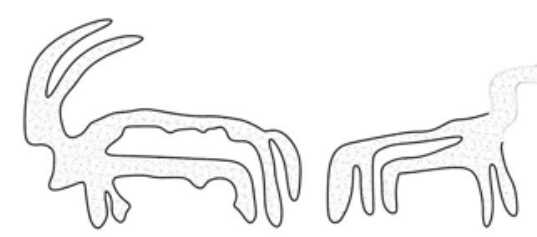

wosks

Figura $1 b$

Esta representación de un cocodrilo probablemente haya que datarla en el periodo Predinástico, tal y como podemos ver en los paralelos hallados en el Alto Egipto ${ }^{14}$ y en la Baja Nubia ${ }^{15}$.

\section{Petroglifo 3}

Tres barcos fluviales Fecha: Naqada IIc

Figuras: $3 a / 3 b$

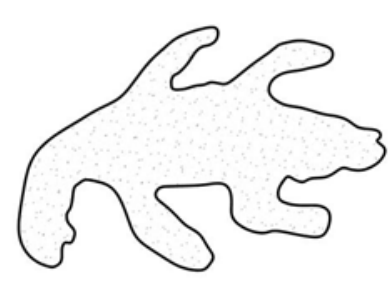

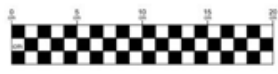

12. Infinidad de ejemplos en Váhala y Červíček, 1999.

13. Montet, 1961: 25.

14. Darnell, 2002: 23, pl. 14.

15. Cf. Hellström, 1970, II: Corpus R; Váhala y Červíček, 1999: 175, 176 no 1. 


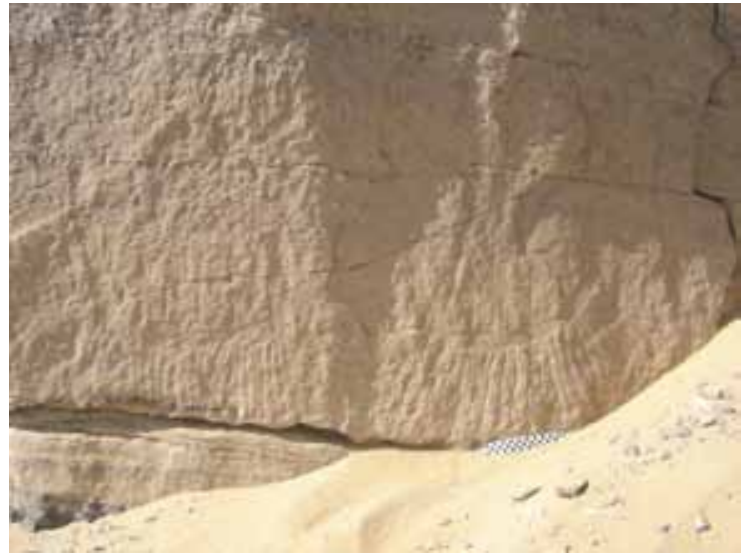

Figura $3 a$

Estas tres barcas se esculpieron en el mismo lienzo de roca, como una serie, si bien parece que fueron hechas en diferentes momentos y por diferentes artistas. Aun así, es casi seguro que las tres se tallaron durante el periodo de Naqada II, es decir a mediados del IV milenio a. C. ${ }^{16}$. Esta precisión cronológica se puede llegar a establecer gracias a los numerosos paralelos encontrados en cerámicas, petroglifos y objetos diversos ${ }^{17}$.

Aunque resulta muy difícil reconstruir la secuencia de la elaboración de estos barcos en relieve, parece probable que la barca central fuera realizada en un primer momento, mientras que las restantes serían talladas en un momento posterior, flanqueando a la primera. La razón principal no es otra que tanto la situada en la izquierda como la de la derecha fueron esculpidas en zonas más rugosas del lienzo de la roca.

Las dos primeras barcas - comenzando por la izquierda- son mucho más grandes y están más

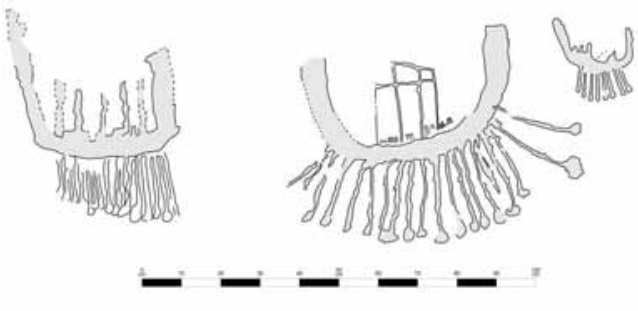

Figura $3 b$

elaboradas que la de la derecha ${ }^{18}$. Ninguna de ellas presenta una figura humana, aunque todas tienen numerosos remos y es posible que se quisiera sugerir con ellos la presencia de remeros.

Otro elemento que parece confirmar la realización en dos fases se basa en la forma de las naves. Aquellas situadas en los extremos tienen un gran parecido a las encontradas en la tumba 100 de Hieracómpolis, actualmente datada en el periodo de Naqada IIc ${ }^{19}$. La barca central recuerda a los ejemplos en cerámicas de esa misma fase predinástica, por lo que las tres pudieron haber sido realizadas durante el ese momento.

Los relieves de estas tres barcas hay que entenderlos dentro del aumento de los contactos entre las diferentes comunidades nilóticas a lo largo del IV milenio a. C. y que sobre todo se multiplicaron desde el periodo de Naqada II $^{20}$. Durante esta fase, las elites emergentes del Alto Egipto demandarían cada vez más y más objetos

16. Hassan, Jiménez-Serrano y Tassie, 2006.

17. Por ejemplo el cuchillo de Gebel el-Arak (Naqada II), el mango de cuchillo del Metropolitan Museun (Naqada II), el lino pintado de Gebelein (Naqada IIc) y la tumba 100 de Hieracómpolis (Naqada IIc).

18. $C f$. la cabina del barco con otros documentados por Hellström, 1970, 2: Corpus V58, y Váhala y Červíček, 1999: 438A no 3,442 no $1,3,9$.

19. Adams y Ciałowicz, 1997: 36-37.

20. Jiménez Serrano, 2003. 


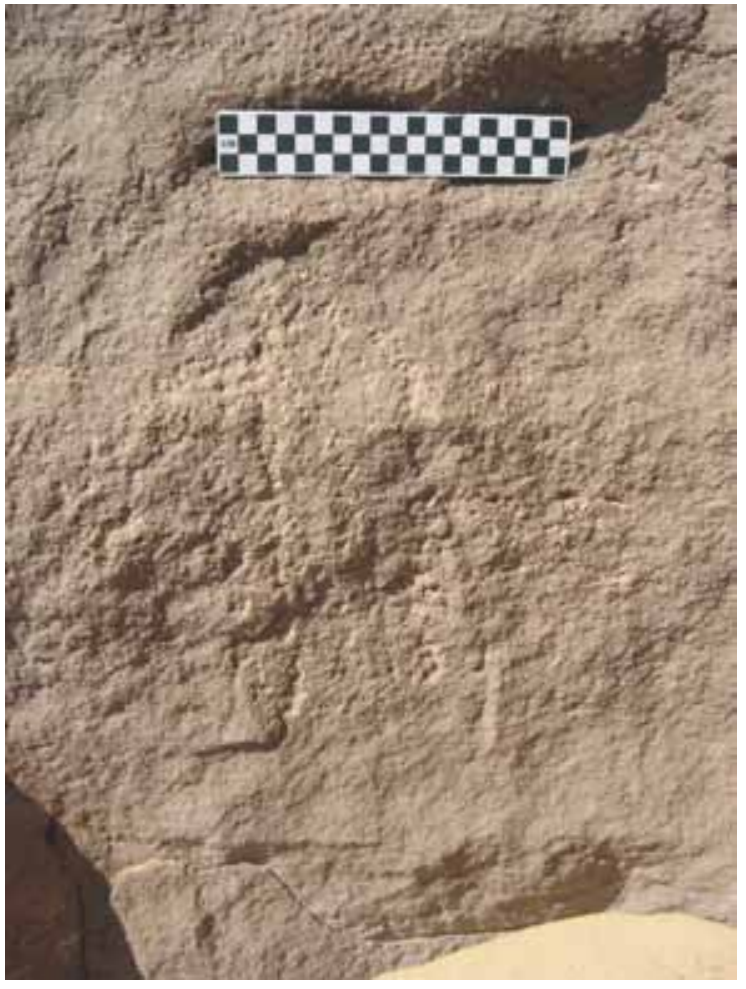

Figura $4 a$

exóticos procedentes de Nubia. De este modo, la más que probable fecha de realización de estas barcas en el periodo de Naqada IIc hay que relacionarlo con la fundación de la cercana Elefantina poco después, en Naqada IId $^{21}$. Es posible que también puedan ser explicada como parte del ciclo solar, tal y como Huyge ${ }^{22}$ propuso no hace mucho.

\section{Petroglifo 4}

[22] ¿Caballo? ¿camello?

Fecha: Primer milenio a. C.-Edad Media

Figuras: 4a / 4b

Resulta difícil poder aseverar qué animal fue representado; esto es, si es un caballo o un camello.

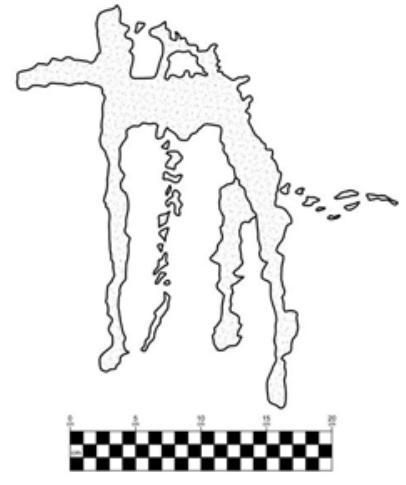

Figura $4 b$

Si se tratara del primero, podría datarse desde el primer milenio a. C. hasta prácticamente la actualidad, aunque la pátina nos indica que no se trata de una obra reciente. Si fuese un camello, interpretación por la que nos inclinamos debido a la longitud de las patas, podría datarse desde finales del primer milenio a. C., momento en el que las tribus blemíes introdujeron este animal en el norte de África.

\section{Petroglifo 5}

Marca antropomorfa

Fecha: desconocida

Figuras: $5 \mathrm{a} / 5 \mathrm{~b}$

No existen paralelos de representaciones como la que aquí presentamos. Ésta recuerda vagamente a 


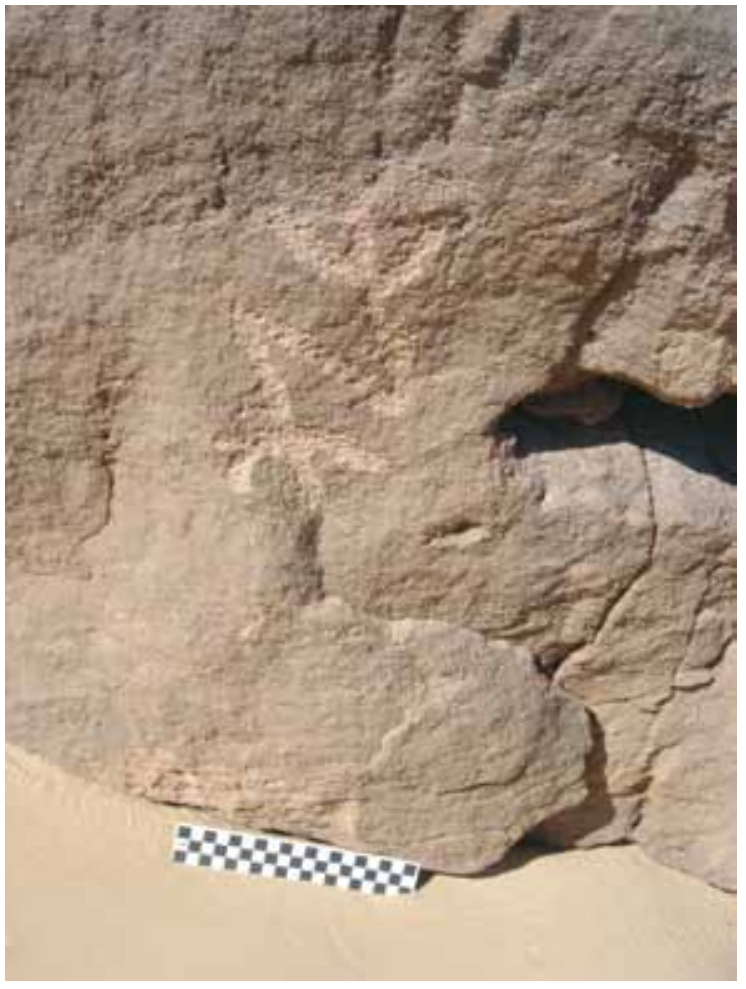

Figura 5 a

las marcas que aparecen relacionadas con canteras ${ }^{23}$ o sobre algunas piedras usadas en la construcción de monumentos, tal vez como alusiones a la fuente original del material ${ }^{24}$. Lo más probable es que haya que identificarla con los símbolos que han sido abundantemente documentados en la Baja Nubia ${ }^{25}$. El buen estado de conservación de grafito indicaría una factura relativamente reciente, si bien cualquier intento de datación no dejaría de ser especulativo.

\section{Inscripciones}

\section{Inscripción 1}

Nombre (i?)

Fecha: Periodo Dinástico ( $i$ ?)

Figuras: 6a / 6b

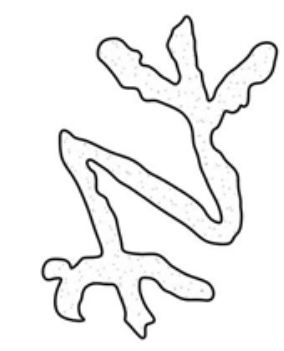

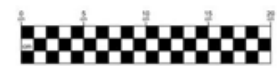

Figura $5 b$

La presente inscripción -si es que en realidad lo es- plantea una serie de problemas debido a la lectura poco clara del hipotético segundo signo. Además, no existen paralelos conocidos de los que podamos a los que podamos recurrir para reconstruir el texto.

El primero de los signos parece representar un cánido, quizá el $(\mathrm{E} 15 \mathrm{~A})^{26}$, lo que lo relacionaría con alguna divinidad como Anubis, Jenty-amentiu o Upuaut. Esta mención nos hace pensar que se trata de un nombre personal, aunque no va acompañado de ningún determinativo de persona. No es necesario insistir en el hecho de que existen numerosos ejemplos en los que el primer elemento onomástico es Anubis ${ }^{27}$.

24. Petrie, 1887: 17.

25. Hellström, 1970, 2: Corpus X.

26. Según la segunda edición de la lista del Center for Computer-Aided Egyptological Research, CCER.

27. Ranke, 1935: $37 \S \S 5-20 ; 1952: 265$ § 7.28. Cf. por ejemplo Allen, 2000: 357-359. 


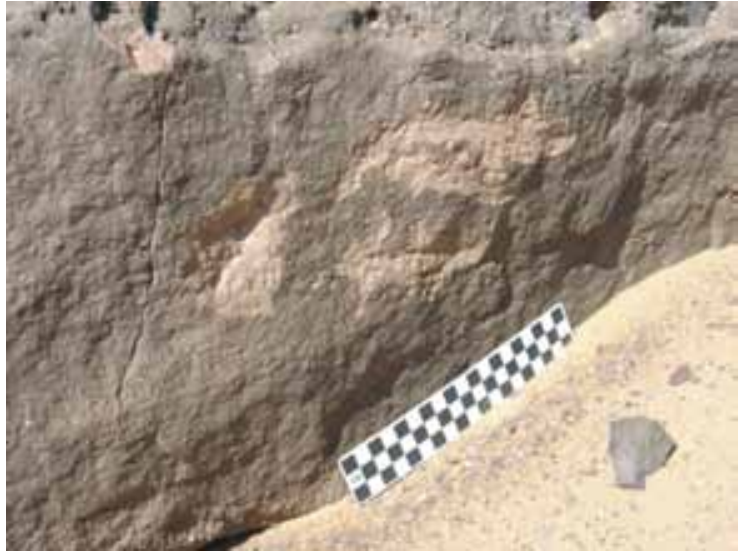

Figura $6 a$

El segundo signo es el que plantea más problemas. Si bien en un principio podría interpretarse como un escorpión, se ha de notar que este signo aparecería orientado en la dirección opuesta a la del signo inmediatamente superior, algo que en principio choca contra los principios de la escritura jeroglífica. La forma recuerda también las barcas fluviales, por lo que haría referencia a algún tipo de festival, pero no hay evidencias de culto a Anubis en la zona y mucho menos de fiestas dedicadas a este dios.

Otra posibilidad es que fuera una representación protectora de una zona usada con fines funerarios.

\section{Inscripción 2}

Inscripción funeraria de Dedu-cheni y de CheniIui

Fecha: Reino Medio (?)

[24] Figura: 7a

La presente inscripción fue descubierta sobre la entrada de una cavidad que parece ser una tumba,

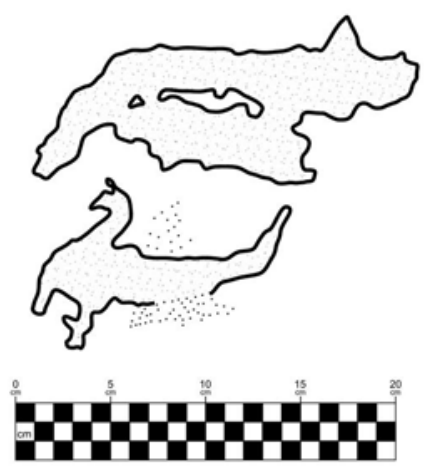

Figura $6 b$

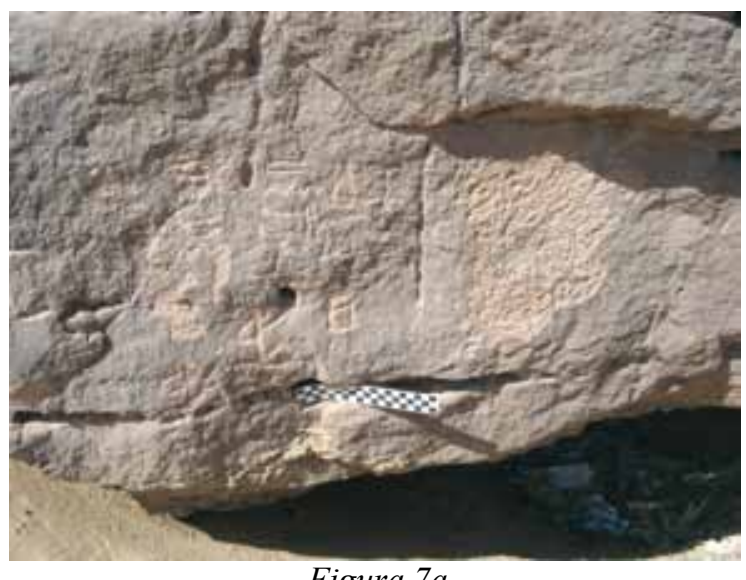

Figura $7 a$

tal y como podría confirmar la existencia de una oquedad cubierta de arena y la presencia de, al menos, un esqueleto humano.

En lo que respecta a la inscripción, se trata de la conocida fórmula de $h t p d j n s w^{28}$. En este caso, el beneficiario sería Dedu-Cheni, nombre atestiguado durante el Reino $\mathrm{Medio}^{29}$. La grafía del nombre no está clara por su mal estado de conservación, podría ser

28. $C f$. por ejemplo Allen, 2000: 357-359.

29. $C f$. Ranke, 1935, I: 403, § 20, que recoge una inscripción del Reino Medio o Segundo Periodo Intermedio en el que aparece el nombre en una inscripción en Kumna, $c f$. Dunham y Janssen, 1960: 166. $C f$. también nombres similares en la Baja Nubia datados en Reino Medio en Žába, 1974: $D d w$, inscr. 41, 48, 153, 168; $D d w$ - $B 3 y$, inscr. 58; $D d w$-Sbk, inscr. 36, 59, 215, 131. 
que coincidiría con una figurilla antropomorfa hallada en Qubbet el-Hawa ${ }^{30}$, o este caso igual a la hallada en Kumna (Baja Nubia $)^{31}$. Nos decantamos por la primera lectura, ya que parece clara la existencia del signo $w$.

La inscripción presenta algunas lagunas provocadas por la acción antrópica y por la erosión natural debida a la ya mencionada debilidad de la roca. Esta última ha afectado principalmente a la segunda y cuarta columnas. Es posible que en éstas se mencionase el nombre de otro difunto $(i ?)$ o bien de quien llevase a cabo el enterramiento. Gracias a los paralelos epigráficos contemporáneos ${ }^{32}$ se podría reconstruir el nombre de $J w j-\underline{t} n j^{33}$, sin duda de sexo masculino por el determinativo.

Frente a la inscripción se han constatado evidencias de una destrucción premeditada (¿damnatio memoriae?), que, originalmente, podría representar alguna ceremonia de presentación del difunto a un determinado dios o bien una escena de ofrendas.

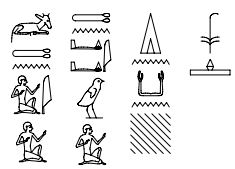

$$
\begin{aligned}
& h t p \text { dj } n s w n k \text { k } n \text {... Ddw-țnj Jwj-țnj }
\end{aligned}
$$

Una excavación de la tumba nos aportaría sin duda una imagen mucho más completa desde el punto de vista histórico. Pese a que no encontramos restos cerámicos que nos permitiesen concretar el periodo, podemos aventurar que se trata de una sepultura de finales del Reino $\mathrm{Medio}^{34} \mathrm{o}$ del Segundo Periodo Intermedio. Para ello, nos basamos principalmente en la localización de la tumba, al sur de Qubbet el-Hawa, zona que en esos periodos ya se encontraría bastante saturada de hipogeos, así como en la onomástica de los difuntos.

La posibilidad de que existan al menos dos personas enterradas en la sepultura era algo común en Qubbet el-Hawa, ya que se trataban más de panteones familiares que de tumbas individuales ${ }^{35}$.

\section{Inscripción 3}

Inscripción: ампа

Fecha: periodo copto

Figuras: $8 \mathrm{a} / 8 \mathrm{~b}$

Es posible que la presente inscripción deba ser leída como дмпа, una variedad dialectal de גחג (si no es esto lo que realmente está escrito). Como es bien sabido, дпג era un título de respeto de la época copta ${ }^{36}$. Resulta curioso el signo que se ha utilizado para la $\Pi$,

30. Edel, 2008: 1293 nr. 0620.01 Fig. 14. La figurilla fue encontrada en el estrato inferior ante la entrada de la tumba QH33 y en ella se puede leer la siguiente inscripción jm3hh(jj) hr Ptḥ Dw-tnj ms(j) Sb.

31. Dunham y Janssen, 1960: 166, Pl. 112 c, R. I. K.

32. Žába, 1974: Paleography E9, publica un paralelo exactamente igual al nuestro.

33. Aunque este nombre no lo recoge Ranke, existen numerosos ejemplos paralelos, $c f$. Ranke, 1935, I: 13, §§12, 26; 16 $\S \S 8-22$.

34. Edel, 2008: 1292 data la estatuilla encontrada ante la entrada de la tumba QH93 a comienzos del Reino Medio. $C f$. también nota 29.

35. Cf. por ejemplo el caso de la familia de Sobek-hotep enterrada en la tumba QH 88, Höveler-Müller, 2006.

36. Crum, 1939: 13. 


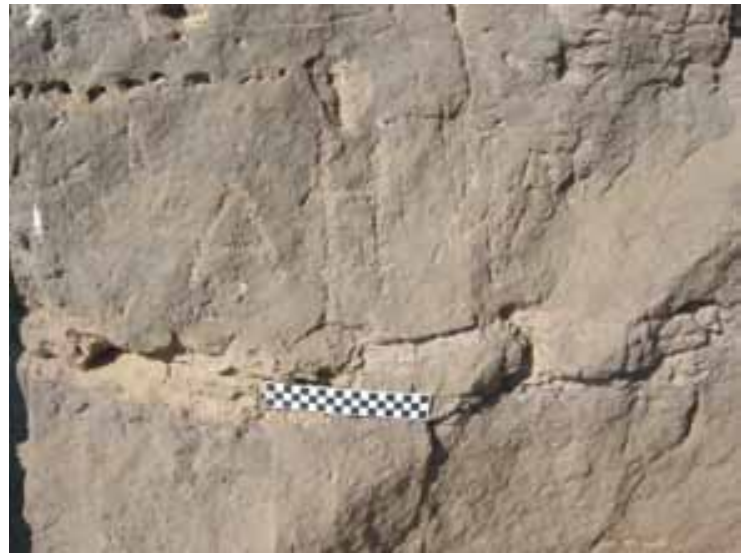

Figura $8 a$

de cuyo diseño no hemos encontrado otro paralelo, aunque sabemos que las inscripciones cristianas de la zona presentan en algunos casos diseños irregulares en ciertas letras ${ }^{37}$. Es posible que esta inscripción esté relacionada con el gran número de visitas que los fieles coptos realizaron a las ruinas del monasterio de San Simeón ${ }^{38}$ pero también puede ser simplemente una referencia escrita inacabada para mayor gloria del obispo local Amba Hatre (finales del s. IV).

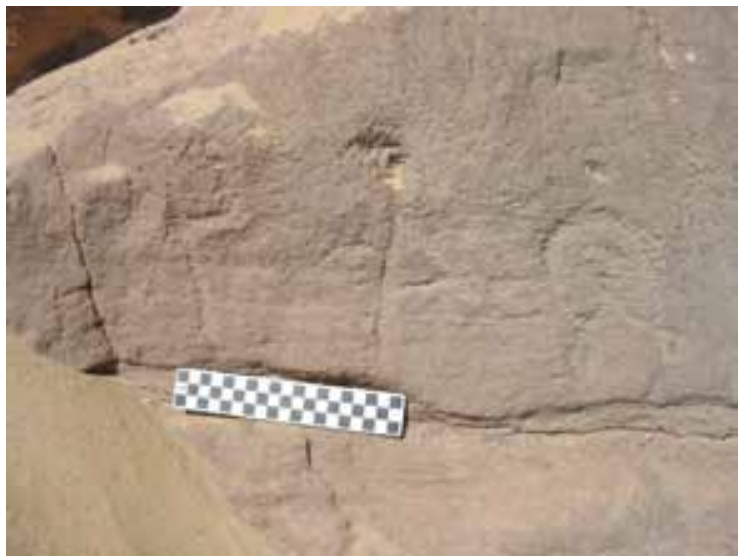

Figura 9a
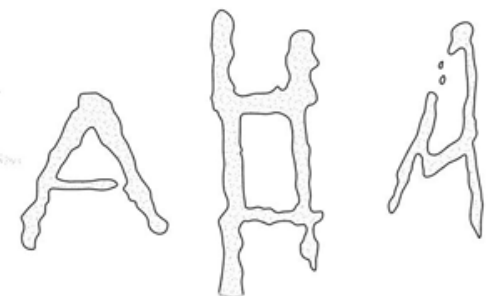

\section{Who}

Figura $8 b$

\section{En catalogación}

\section{Documento 1}

Fecha: Desconocida

Figuras: 9a / 9b

Se trata de una inscripción de difícil catalogación e interpretación. Los tres signos podrían considerarse, en principio, como jeroglíficos, si bien su lectura es desconocida. Por otro lado, los dos primeros,

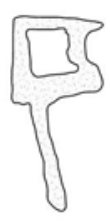

Figura $9 b$

37. $C f$. los numerosos ejemplos en De Morgan et alii, 1894: 140.

38. De Morgan et alii, 1894: 138-139.
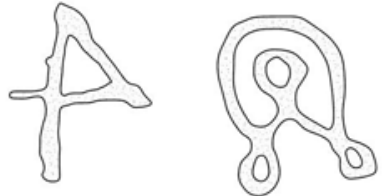

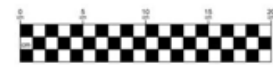




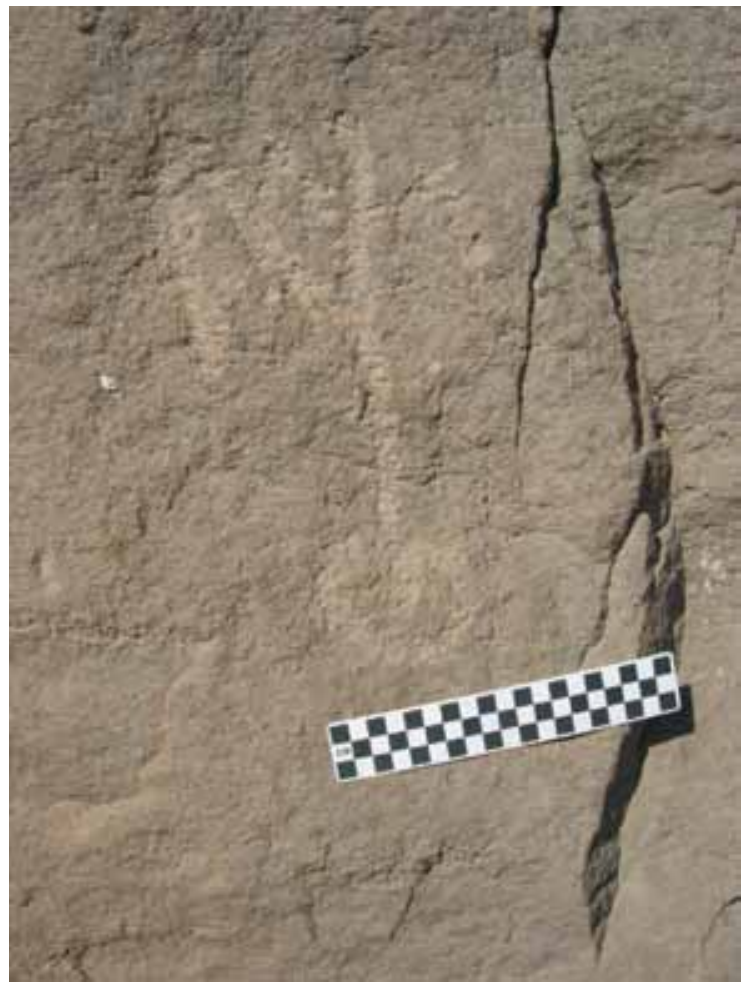

Figura 10a

comenzando por la izquierda, podrían ser también coptos (pd), mientras que el tercero sería el dibujo de un objeto presente en al menos tres grafitos en la Baja Nubia e interpretado como un ornamento ${ }^{39}$.

\section{Documento 2}

Fecha: desconocida

Figuras: 10a / 10b

No se ha encontrado ningún paralelo con la presente figura.

3. TRASCENDENCIA DE LOS DESCUBRIMIENTOS Y FUTURAS ACTUACIONES

El hecho de que aún se puedan encontrar nuevas fuentes históricas en un yacimiento en teoría tan

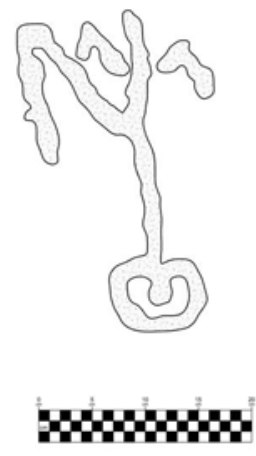

Figura $10 b$ explotado como Garb Asuán confirma -una vez más- la gran potencialidad arqueológica de Egipto. Nuestro trabajo aquí presentado sigue la línea de otros que en los últimos años se están centrando en la investigación de áreas periféricas, relegadas por muchos investigadores en épocas pasadas.

La heterogeneidad cronológica de los grafitos hallados nos muestra la importancia de la región desde la Prehistoria reciente, revelando en algunos casos - como en el de los barcos de Naqada II (Fig. 3) - la gran importancia de la región en las comunicaciones entre Egipto y Nubia, fruto de la cual se materializará la fundación de Elefantina. Es posible que otros no hayan sido descubiertos por la acción conjunta del viento y la arena, aunque sospechamos que el número no sería muy elevado ya que la mala calidad de la piedra en Garb Asuán Sur no invita a tallarla.

39. Cf. Váhala y Červíček, 1999: no 3301-303, Taf. 77. 
Hasta los trabajos de investigación de nuestra misión era bien conocido el uso de Qubbet el-Hawa como necrópolis de los altos funcionarios al menos desde finales del Reino Antiguo. Actualmente, gracias a nuestras prospecciones, confirmadas por los análisis de georádar, sabemos que hay al menos otras dos zonas de enterramiento, una prácticamente destruida y otra aún por evaluar, si bien los indicios muestran un panorama bastante esperanzador. Como ya avanzábamos, es posible que, al menos una tumba date de finales del Reino Medio o del Segundo Periodo Intermedio. Todo ello resultaría de gran interés, pues nuestro conocimiento de la historia local de Asuán en esa época resulta muy escaso. A parte de la que ya hemos avanzado anteriormente, no queremos obviar la presencia de restos de diques de atraque a los pies de las tumbas y que darían paso a rampas de acceso -actualmente bajo la arena-como las que se pueden ver en Qubbet el-Hawa.

Por otro lado, los citados análisis de georádar, han permitido localizar el posible emplazamiento de varias tumbas en la necrópolis de Qubbet el-Hawa, algunas de ellas de grandes dimensiones.

En definitiva, esperamos que en los próximos años los frutos de nuestro trabajo tengan como resultado la conservación, la puesta en valor y el mejor conocimiento del yacimiento principal de Garb Asuán Sur, Qubbet el-Hawa. Para conocer más detalles de las dos nuevas necrópolis descubiertas, habrá que esperar. 


\section{Bibliografía}

Adams, B.; Cialowicz, K. M.

1997 Protodynastic Egypt. Pembrokeshire.

Al-Khouri, M.

2005 Gharbi Aswan: A research for conservation of the mural paintings, en Al-Khouri, M. et alii (eds.): Archaeology and Conservation in the Middle East, Roma: 188-199.

Allen, J. P.

2000 Middle Egyptian. Cambridge.

ČERVíČEK, P.

1974 Felsbilder des Nord-Etbai, oberägyptens und unternubiens. Wiesbaden.

Crum, W. E.

1939 A Coptic Dictionary. Oxford.

DARNELL, J. C.

2002 Theban Desert Road Survey in the Egyptian Western Desert. Vol. 1. Gebel Tjauti Rock Inscriptions 1-45 and Wadi el-Hôl Rock Inscriptions 1-45. Chicago.

Dunham, D.; Janssen, J. M. A. 1960 Semna. Kumma. Boston.

EDEL, E.

2008 Die Felsgräbernekropole der Qubbet elHawa bei Assuan. München, 3 vols.

HABACHI, L.

1981 Sixteen Studies on Lower Nubia. Cairo.

Hassan, F. A.; Jiménez-Serrano, A.; Tassie, G. A. 2006 The Sequence and Chronology of the Protodynastic and Dynasty I Rulers, en Kroeper, K.; Chlodnicki, M. y Kobusiewicz, M. (eds.): Archaeology of Early Northeastern Africa. In Memory of Lech Krzyzaniak, (SAA 9), Poznan: 687-722.
Heldal, T.; Bøe, R.; Müller, A.

2007 Geology and stone resources of the Aswan West Bank, en Bloxam, E.; Heldal, T. y Storemyr, P. (eds.): QuarryScapes Report. Characterisation of complex quarry landscapes: an example from the West Bank quarries, Aswan, The Geological Survey of Norway: 51-68.

HellströM, P.

1970 The Rock Drawings. Odense, 2 Vols.

HÖVELER-MÜLLER, M.

2006 Funde aus den Grab 88 der Qubbet elHawa bei Assuan. Wiesbaden.

Huyge, D.

2002 Cosmology, Ideology and Personal Religious Practice in Ancient Egyptian Rock Art, en R. Friedman (ed.): Egypt and Nubia. Gifts of the Desert, London: 192-206.

JimÉnez Serrano, A.

2003 Two Protokingdoms in Lower Nubia at the End of the Fourth Millennium BC, en Krzyżaniak, L.; Kroeper, K. y Kobusiewicz, M. (eds.): Cultural Markers in the Later Predistory of Northeastern Africa and Recent Research, (SAA 8), Poznan: 251268.

2004 Elephants standing on Hills or the Oldest Name of Elephantine, en Hendrickx, S. y Friedman, R.F. (eds.): Egypt at its Origins. Studies in Memory of Barbara Adams. Proceedings of the International Conference "Origin of the State. Predynastic and Early Dynastic Egypt", Krakow, 28th August - 1st September 2002, (OLA 138), Louvain: 847-858.

Kopr, $\mathrm{P}$.

2006 Elephantine XXXII. Die Siedlung der Naqadazeit. Mainz am Rhein. 
Morgan, J. DE ET ALII

1894 Catalogue des Monuments et Inscriptions de l'Egypte Antique. Première Série. Haute Égypte. Tome Premier. De la Frontière de Nubie a Kom Ombos. Wien.

Montet, P.

1961 Géographie de l'Égypte Ancienne. La Haute Égypte. Paris.

Petrie, W. M. F.

1887 A Season in Egypt. London.

RANKE, $\mathrm{H}$.

1935-1977 Die Ägyptischen Personennamen. Glückstadt, 3 vols.
VÁHALA, F.; ČERvíČEK, P.

1999 Katalog der Felsbilder aus der Tschecholslowakischen Konzession in $\mathrm{Nu}$ bien. Praga.

Weigall, A. E. P.

1910 Guide to the Antiquities of Upper Egypt, from Abydos to the Sudan Frontier. London.

ŽÁBA, Z.

1974 The Rock Inscriptions of Lower Nubia. Czechoslovak Concession. Prague. 


\section{Trabajos de Egiptología Papers on Ancient Egypt}

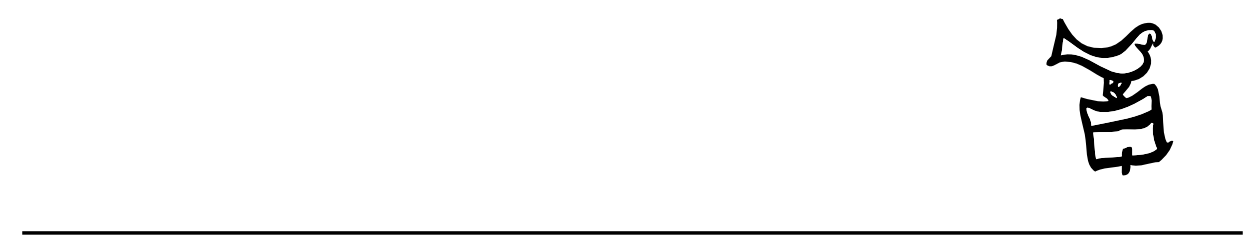

Número 5/2 2009 


\section{Actas \\ III Congreso Ibérico de Egiptología III Congresso Ibérico de Egiptologia}

Editores

Miguel Ángel Molinero Polo Covadonga Sevilla Cueva 


\title{
Editor
}

Miguel Ángel Molinero Polo

Universidad de La Laguna

\section{Consejo Editorial}

\author{
Antonio Pérez Largacha \\ Universidad de Castilla-La Mancha \\ José-R. Pérez-Accino \\ Birkbeck, Universidad de Londres \\ Covadonga Sevilla Cueva \\ Universidad Autónoma de Madrid
}

\section{Comité Científico}

Josep Cervelló i Autuori

Universitat Autònoma de Barcelona

$\mathrm{M}^{\mathrm{a}}$ José lópez Grande

Universidad Autónoma de Madrid

Josep Padró i Parcerisa

Universitat de Barcelona

$\mathrm{M}^{\mathrm{a}}$ Carmen Pérez Die

Museo Arqueológico Nacional, Madrid

Ester Pons Mellado

Museo Arqueológico Nacional, Madrid

José M. Serrano Delgado

Universidad de Sevilla

\section{Colaboradores Editoriales}

Linda Steynor

English editorial assistant

Hervé Mouriacoux

Assistant éditorial pour la langue française 
Trabajos de Egiptología está producida por Isfet. Egiptología e Historia c/ Blanco $1,2^{\circ}$

38400 Puerto de la Cruz

Tenerife-Islas Canarias

España

Maquetación: Proyecto Limón

(C) Autores de los artículos aparecidos

y Consejo Editorial de Trabajos de Egiptología - Papers on ancient Egypt

Depósito Legal: TF-2303-2009

ISSN: $1695-4750$

Imprime: Gráfica Los Majuelos, S.L.L.

imprenta@graficaslosmajuelos.com

Tfno.: 922311455 


\section{Comité Científico \\ III Congreso Ibérico de Egiptología III Congresso Ibérico de Egiptologia}

Miguel Á. Molinero Polo

Universidad de La Laguna

Presidente del Comité Organizador del III Congreso Ibérico de Egiptología

Miembro del Comité Organizador del I Encuentro de Egiptología

Josep Cervelló Autuori

Universitat Autònoma de Barcelona

Presidente del Comité Organizador del II Congreso Ibérico de Egiptologia

José Manuel Galán Allué

Consejo Superior de Investigaciones Cientificas

Director del Proyecto Djehuty, Luxor, Egipto

$\mathrm{M}^{\mathrm{a}}$ Helena Trindade Lopes

Universidad de Lisboa

Directora de la Misión Arqueológica Portuguesa en Menfis

Josep Padró i Parcerisa

Universitat de Barcelona

Director de la Misión Arqueológica de Oxirrinco

Antonio Pérez Largacha

Universidad de Castilla - La Mancha

Miembro del Comité Organizador del I Encuentro de Egiptología

José Ramón Pérez-Accino

Birkbeck College, University of London

Miembro del Comité Organizador del I Encuentro de Egiptología

$\mathrm{M}^{\mathrm{a}}$. Carmen Pérez Díe

Museo Arqueológico Nacional

Directora de la Misión Arqueológica Española en Heracleópolis Magna, Egipto

Covadonga Sevilla Cueva

Universidad Autónoma de Madrid

Miembro del Comité Organizador del I Encuentro de Egiptología 